\title{
DESIGN OF NON-ANTHROPOMORPHIC ROBOTIC HANDS FOR ANTHROPOMORPHIC TASKS
}

\author{
Edgar Simo-Serra \\ Institut de Robòtica i Informàtica Ind. \\ (CSIC-UPC) \\ Llorens i Artigas, 4-6 \\ 08028 Barcelona, Spain \\ Email: esimo@iri.upc.edu
}

\author{
Francesc Moreno-Noguer \\ Institut de Robòtica i Informàtica Ind. \\ (CSIC-UPC) \\ Llorens i Artigas, 4-6 \\ 08028 Barcelona, Spain \\ Email: fmoreno@iri.upc.edu
}

\author{
Alba Perez-Gracia* \\ Institut de Robòtica i Informàtica Ind. \\ (CSIC-UPC) \\ Llorens i Artigas, 4-6 \\ 08028 Barcelona, Spain \\ And: College of Science and Engineering \\ Idaho State University \\ Pocatello, Idaho 83209 \\ Email: aperez@iri.upc.edu
}

\begin{abstract}
In this paper, we explore the idea of designing nonanthropomorphic multi-fingered robotic hands for tasks that replicate the motion of the human hand. Taking as input data a finite set of rigid-body positions for the five fingertips, we develop a method to perform dimensional synthesis for a kinematic chain with a tree structure, with five branches that share three common joints.

We state the forward kinematics equations of relative displacements for each serial chain expressed as dual quaternions, and solve for up to five chains simultaneously to reach a number of positions along the hand trajectory. This is done using a hybrid global numerical solver that integrates a genetic algorithm and a Levenberg-Marquardt local optimizer.

Although the number of candidate solutions in this problem is very high, the use of the genetic algorithm allows us to perform an exhaustive exploration of the solution space to obtain a set of solutions. We can then choose some of the solutions based on the specific task to perform. Note that these designs match the task exactly while generally having a finger design radically different from that of the human hand.
\end{abstract}

\section{NOMENCLATURE}

$b$ Number of kinematic chains branches.

$n$ Number of joints.

$m$ Number of end-effector positions for all branches considered.

$\varepsilon \quad$ Dual unit such that $\varepsilon^{2}=0$. $\mathbf{v}$ A vector.

$\hat{Q} \quad$ A dual quaternion.

$\mathrm{S}$ Plücker coordinates of a line: $\mathrm{S}=\mathbf{S}+\varepsilon \mathbf{s}^{0}$

\section{INTRODUCTION}

There are many applications for which a robotic system is needed to work in human environments and to perform tasks that are designed for the human hand. In most cases, the solution adopted for grasping and manipulation consists of anthropomorphic robotic hands, which imitate to a certain extent the topology and joint location of the human hand. See [1] for a review of applications and concept definition.

It is difficult to match the complexity of the human hand, commonly accepted to have 26 degrees of freedom when counting the motion at the wrist and the pronation/supination of the forearm. The anthropomorphic design must include a complex mechanical system, actuation and sensing in a small space [2]. In order to reduce complexity, current designs limit the active degrees of freedom, through simplification of the mechanical structure or by designing underactuated hands. The design of simplified grippers limits the tasks of the robotic end-effector to some grasping and manipulation actions. Pairing these designs with some degree of underactuation and compliance, it is then possible to perform robust grasping of objects of unknown shape. Dollar and Howe [3] present a simplified, underactuated design for reliable grasping. Ciocarlie and Allen [4] optimize an underactuated, non-anthropomorphic gripper for performing a series of grasps from a database. A more thorough review on underactuated hands can be found in [5].

In order to perform some of the more complex functions

*Address all correspondence to this author. 
of the human hand (not only grasping and manipulation, but also perception through surface exploration), it seems that an end-effector with several independently-actuated fingers may be needed. There are many examples of anthropomorphic robotic hands, see for instance [6] for a relatively recent review. However, for complex multi-fingered designs, it may not be necessary that the robotic fingers mimic those of the human hand in order to perform human-like tasks. We want to explore these alternative designs with the use of kinematic synthesis.

These alternate designs may have multiple applications. One possible usage is on the design of exoskeletons. These exoskeletons move the finger tips exactly like a human hand, while being able to be mounted on a hand without interfering with the hand's movement. Another application is the improvements of existing designs. Solutions can be chosen with additional criteria like equal distribution of joint velocities for a given task or location criteria for the joint axes. This can allow, for instance, designs optimized for particular actuators.

Kinematic design of robotic hands has focused on the design of individual motion of fingers or parts of the hand. Dai and Wang [7] use kinematic synthesis to design a spherical mechanism to act as the palm of a metamorphic hand. Van Varseveld and Bone [8] designed a finger mechanism for a nonanthropomorphic dexterous hand. Walker et al. [9] also design planar linkages for the fingers of a non-anthropomorphic, dexterous hand. Schafer and Dillman [10] present the kinematic design of a humanoid robotic wrist.

In this paper, we develop a method for the design of a full non-anthropomorphic multi-fingered robotic hand for tasks that replicate the motion of the human hand at the fingertips. Taking as input data a finite set of rigid-body positions for the human fingertips, we perform dimensional synthesis for a kinematic chain with a tree structure, with three common joints and five branches. As a whole, the process entails the simultaneous solution of up to five serial chains, two of them having four independent joints and three of them with five independent joints, plus three common joints for all of them. The total degrees of freedom of the non-anthropomorphic hand design is 26 , similar to the human hand.

We state the forward kinematics equations of relative displacements for each serial chain expressed as dual quaternions, and solve for all five chains simultaneously to reach a number of positions along the hand trajectory. The synthesis of spatial serial chains for up to five degrees of freedom using this technique was developed in [11]. We use a similar methodology together with a hybrid global numeric solver, composed of a genetic algorithm paired with a Levenberg-Marquardt local optimizer. For the tree-like kinematic structure, a high number of positions can be defined to perform exact synthesis, obtaining a good approximation for the desired trajectory.

Finding the complete solution set for the synthesis of complex kinematic chains is an unsolved problem. Only dyads, such

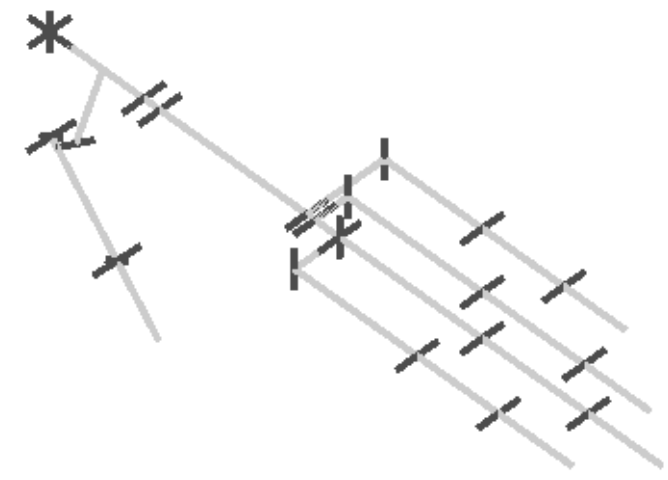

FIGURE 1: HAND SKELETON USED TO GENERATE POSITIONS.

as the RR kinematic chain $[12,13]$, and some triads with particular characteristics [14], have been fully studied. Even the 3R kinematic chain has not been completely solved with a closed algebraic expression [15]. The complexity of a tree-like kinematic chain with a total of 26 revolute joints, and our numerical results, lead to believe that there will be a very large amount of solutions. This is an issue that has been found before even for simpler kinematic chains, see [14]. In order to deal with this, additional constraints may be added to help in the selection of the final design, depending on the task. We present two of the solutions for a hand task that was synthetically generated. The designs exactly match the task while having a finger design radically different from that of the human hand.

\section{KINEMATIC HAND MODEL}

The human hand has 5 fingers formed by 14 joints. These joints are created at the contact surface of the 27 major bones forming the hand. The joints do not exactly match the motion of a classical lower pair, and some of them present more than one degree of freedom. However, they can be modeled fairly precisely using only simple revolute kinematic joints. The axes of these revolute joints do not necessarily have to intersect within the same joint.

The full hand can be modeled using a total of 26 joints if we consider the wrist and the pronation/supination of the forearm to be formed by 3 revolute joints. The index and middle finger have 4 revolute joints, while the third, fourth and thumb have 5 revolute joints. The dimensions are taken from the literature [16, 17]. The skeleton can be represented by drawing the common normals of the joint axes as shown in Fig. 1. 
TABLE 1: JOINT ANGLES USED TO GENERATE THE TRAJECTORY.

\begin{tabular}{cl}
\hline Chain & Hand task revolute joint limits $\left[\theta_{\min }-\theta_{\max }\right]$ \\
\hline Common & {$\left[-90^{\circ}, 90^{\circ}\right],\left[-90^{\circ}, 90^{\circ}\right],\left[-90^{\circ}, 90^{\circ}\right]$} \\
Index & {$\left[-10^{\circ}, 90^{\circ}\right],\left[-10^{\circ}, 10^{\circ}\right],\left[0^{\circ}, 100^{\circ}\right],\left[0^{\circ}, 90^{\circ}\right]$} \\
Middle & {$\left[-10^{\circ}, 90^{\circ}\right],\left[-10^{\circ}, 10^{\circ}\right],\left[0^{\circ}, 100^{\circ}\right],\left[0^{\circ}, 90^{\circ}\right]$} \\
Third & {$\left[-10^{\circ}, 10^{\circ}\right],\left[0^{\circ}, 20^{\circ}\right],\left[-10^{\circ}, 10^{\circ}\right],\left[0^{\circ}, 100^{\circ}\right],\left[0^{\circ}, 90^{\circ}\right]$} \\
Fourth & {$\left[-10^{\circ}, 10^{\circ}\right],\left[0^{\circ}, 20^{\circ}\right],\left[-10^{\circ}, 10^{\circ}\right],\left[0^{\circ}, 100^{\circ}\right],\left[0^{\circ}, 90^{\circ}\right]$} \\
Thumb & {$\left[-25^{\circ}, 25^{\circ}\right],\left[-25^{\circ}, 25^{\circ}\right],\left[-10^{\circ}, 10^{\circ}\right],\left[0^{\circ}, 70^{\circ}\right]$,} \\
& {$\left[-10^{\circ}, 85^{\circ}\right]$} \\
\hline
\end{tabular}

\section{Hand Task Generation}

The task is defined as a series of finite positions (locations and orientations) for each finger tip, created by assigning a set of joint variables to the kinematic model. The range of motion of each joint varies greatly among the population and it is therefore impossible to assign a precise range to each joint. In this paper, we will evaluate our solver using synthetically generated trajectories. These trajectories are generated from random positions within the joint angle ranges defined in Table 1 to simplify the convergence of the solver.

\section{KINEMATIC SYNTHESIS}

The goal of the dimensional kinematic synthesis is to find the location and orientation of a set of joint axes able to perform a given motion, where the number and type of joints are pre-defined. This is also known as the motion-to-form problem in which we are given a motion, defined by a sequence of endeffector positions, as an input and must calculate the form as a set of joints and angles that can perform the motion. In this paper, we follow the original idea of [18] of using the forward kinematics equations of the kinematic chain, but formulated as relative displacements and expressed as dual quaternions, see [11] for a complete description of this approach.

The input data for the synthesis are the $m-1$ relative transformations $\hat{P}_{1 j}=\cos \frac{\Delta \hat{\phi}_{1 j}}{2}+\sin \frac{\Delta \hat{\phi}_{1 j}}{2} \mathrm{P}_{1 j}, j=2, \ldots, m$, defining the task; the output are the Plücker coordinates $S_{i}=\mathbf{s}_{i}+\varepsilon \mathbf{s}_{i}^{0}=$ $\mathbf{s}_{i}+\varepsilon \mathbf{c}_{i} \times \mathbf{s}_{i}, i=1, \ldots, n$, of the $n$ joints that define the kinematic chain at a reference configuration, and also the $j=2, \ldots, m$ sets of joint variables $\boldsymbol{\Delta} \hat{\boldsymbol{\theta}}_{j}=\theta_{i j}-\theta_{i 1}+\varepsilon\left(d_{i j}-d_{i 1}\right), i=1, \ldots, n$, used to reach the task positions, measured from the reference configuration $\hat{\boldsymbol{\theta}}_{1}=\theta_{i 1}+\varepsilon d_{i 1}, i=1, \ldots, n$.

\section{Forward Kinematics}

We can represent each individual branch of a tree-like topology as an individual kinematic serial chain, which shares a number of joints with other branches. Given a kinematic serial chain with $n$ joints, we can write the kinematics equations using the product of exponentials of the screws corresponding to the joint axes, as described in [19]. In this paper, instead of calculating the exponentials using matrix algebra, we do the exponentials for the Clifford even subalgebra of the projective space, in which the unit elements, also known as dual quaternions, express spatial displacements. The exponential of a screw represented by the Clifford algebra element $\mathrm{J}=(1+\mu \varepsilon) \mathrm{S}$ of axis $\mathrm{S}$, where $\mu$ is the pitch relating the slide $d$ and the rotation $\theta$ along and about the screw, yields a finite displacement,

$$
\begin{aligned}
e^{\frac{\theta}{2} J} & =\left(\cos \frac{\theta}{2}-\frac{d}{2} \sin \frac{\theta}{2} \varepsilon\right)+\left(\sin \frac{\theta}{2}+\frac{d}{2} \cos \frac{\theta}{2} \varepsilon\right) S \\
& =\cos \frac{\hat{\theta}}{2}+\sin \frac{\hat{\theta}}{2} \mathrm{~S} .
\end{aligned}
$$

For a serial chain with $n$ joints, in which each joint can rotate an angle $\theta_{i}$ and slide a distance $d_{i}$, around and along the axis $S_{i}$, $i=1, \ldots, n$, we calculate the forward kinematics of relative displacements (with respect to an arbitrary reference configuration),

$$
\begin{aligned}
\hat{Q}(\boldsymbol{\Delta} \hat{\boldsymbol{\theta}}) & =e^{\frac{\Delta \hat{\theta}_{1}}{2} \mathrm{~S}_{1}} e^{\frac{\Delta \hat{\theta}_{2}}{2} \mathrm{~S}_{2}} \cdots e^{\frac{\Delta \hat{\theta}_{n}}{2} \mathrm{~S}_{n}} \\
& =\left(\cos \frac{\Delta \hat{\theta}_{1}}{2}+\sin \frac{\Delta \hat{\theta}_{1}}{2} \mathrm{~S}_{1}\right) \cdots\left(\cos \frac{\Delta \hat{\theta}_{n}}{2}+\sin \frac{\Delta \hat{\theta}_{n}}{2} \mathrm{~S}_{n}\right),
\end{aligned}
$$

where $\boldsymbol{\Delta} \hat{\boldsymbol{\theta}}=\boldsymbol{\theta}_{j}-\boldsymbol{\theta}_{1}+\boldsymbol{\varepsilon}\left(\mathbf{d}_{j}-\mathbf{d}_{1}\right)$ contains the joint variables, as relative values with respect to the joint parameters of the chain $\boldsymbol{\theta}_{1}$ and $\mathbf{d}_{1}$ when in the reference configuration.

\section{Synthesis Design Equations}

The dimensioning of the articulated system has to be done so that the forward kinematics equations in Eqn. (2) can reach all the desired task positions $\hat{P}_{1 j}$,

$$
\hat{P}_{1 j}=e^{\frac{\Delta \hat{\theta}_{1 j}}{2} \mathrm{~S}_{1}} e^{\frac{\Delta \hat{\theta}_{2 j}}{2} \mathrm{~S}_{2}} \cdots e^{\frac{\Delta \hat{\theta}_{n j}}{2} \mathrm{~S}_{n}}, \quad j=2, \ldots, m
$$

where $j=1$ is reserved for the reference configuration.

This results in $8(m-1)$ design equations, considering the fact that we are using dual quaternions which have 8 components. The design variables that determine the dimensions of the 


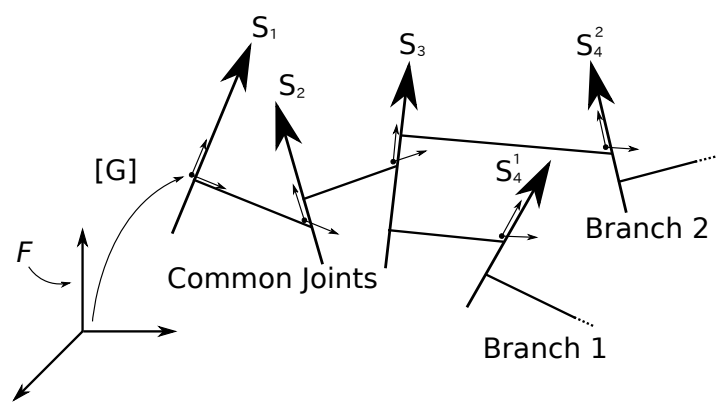

FIGURE 2: TOPOLOGY OF THE KINEMATIC CHAIN.

chain are the $n$ joint axes $S_{i}, i=1, \ldots, n$, in the reference configuration. In addition, the equations contain the $n(m-1)$ pairs of joint parameters $\Delta \hat{\theta}_{i j}=\Delta \theta_{i j}+\varepsilon \Delta d_{i j}$, which are also unknown.

\section{NON-ANTHROPOMORPHIC HAND SYNTHESIS}

In this paper, we are interested in the synthesis of robotic grippers for human-hand tasks. We do not impose any explicit limitation on the link dimensions or placement. The resulting design should be able to perform a given human-hand task while having a non-anthropomorphic aspect.

\section{System Topology}

As input topology, we define a tree-like kinematic chain with three common joints in series at the base, connected to five serial chains arranged in parallel, two of them with four degrees of freedom and three of them with five degrees of freedom. This structure follows the generally accepted joint arrangement of the human hand plus the wrist motion. Figure 2 shows the topology of the kinematic chain to be synthesized.

Note that, to our knowledge, no previous approach has addressed the synthesis of tree-like structures. In addition, tree topologies can also be used to represent pure serial topologies, when there is only one branch, and loop topologies, when endeffectors of multiple branches are placed at the same position.

\section{Design Equations}

We state the design equations by adapting Eqn. (3) to our particular topology. For each one of the serial chains in the parallel arrangement we state a set of design equations, to obtain the total system of equations

$$
\begin{aligned}
& \hat{P}_{1 j}^{k}=e^{\frac{\Delta \hat{\theta}_{1 j}}{2} S_{1}} e^{\frac{\Delta \hat{\theta}_{2 j}}{2} S_{2}} e^{\frac{\Delta \hat{\theta}_{3 j}}{2} S_{3}} e^{\frac{\Delta \hat{\theta}_{j j}^{k}}{2} S_{4}^{k}} \cdots e^{\frac{\Delta \hat{\theta}_{7 j}^{k}}{2} S_{7}^{k}}, \quad k \in\{1,2\},
\end{aligned}
$$

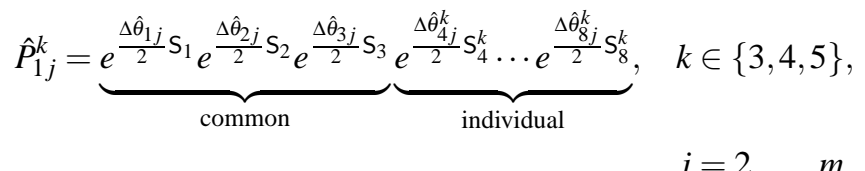

Here $k$ identifies the kinematic chain and $j=2, \ldots, m$ is the index of the task position. The fingers are denoted by: index, $k=1$; middle, $k=2$; third, $k=3$; fourth, $k=4$ and thumb, $k=5$.

Each of the five serial chains, taken individually, has a total of seven or eight degrees of freedom, depending on the finger it corresponds to. Even though the exact dimensional synthesis does not apply to serial chains with six or more degrees of freedom, by having three common degrees of freedom we are able to state the design equations of the system as a whole.

Notice that even though each individual finger has more than six degrees of freedom, the tree-like architecture is not kinematically redundant when defining the motion of several branches at the same time.

\section{KINEMATIC SOLVER}

In order to deal with the large system of equations presented in the previous section, the selected solver aims to minimize the error in Eqn. (4) for each kinematic chain. In principle, problems with selection of the metric does not apply to this case, as we are targeting exact synthesis.

The objective of the solver is to perform general inverse kinematics, that is, adjust both joint angles and joint axes to follow a motion. However, by making the joint axes constant it can also perform inverse kinematics.

\section{Objective Functions}

Given a vector $v=\{4,4,5,5,5\}$ with the number of independent joints for each finger, the design equations from Eqn. (4) can be written as a set of unconstrained functions,

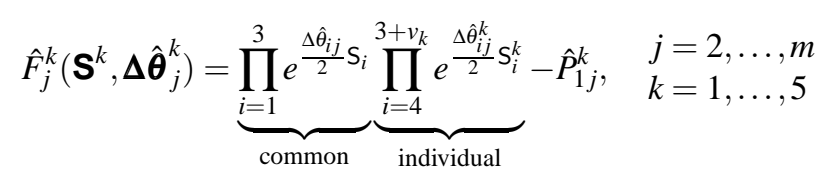

where we can separate the product of the common joints from the individual joints that belong to each branch. 
The global objective of the solver is to solve the design equations defined in Eqn. (4). This can be written as,

$$
\sum_{j=2}^{m} \sum_{k=1}^{5}\left|\hat{F}_{j}^{k}\left(\mathbf{S}^{k}, \boldsymbol{\Delta} \hat{\boldsymbol{\theta}}_{j}^{k}\right)\right|=0
$$

In order to avoid falling into local minima we will use a hybrid solver composed of both a genetic algorithm and a Levenberg-Marquadt optimizer.

Genetic algorithms behave better with defined positive maximization objective functions. We can convert Eqn. (6) to a minimization function and invert it to obtain,

$$
\underset{\mathbf{S}, \Delta \hat{\boldsymbol{\theta}}}{\operatorname{maximize}}\left(\sum_{j=2}^{m} \sum_{k=1}^{5}\left|\hat{F}_{j}^{k}\left(\mathbf{S}^{k}, \boldsymbol{\Delta} \hat{\boldsymbol{\theta}}_{j}^{k}\right)\right|\right)^{-1}
$$

which we can see is defined positive if the domain of Eqn. (5) is finite. This single function is also known as the fitness function.

For the Levenberg-Marquadt local optimizer we use the minimizing least squares objective function,

$$
\underset{\mathbf{S}, \Delta \hat{\boldsymbol{\theta}}}{\operatorname{minimize}} \sum_{j=2}^{m} \sum_{k=1}^{5} \hat{F}_{j}^{k}\left(\mathbf{S}^{k}, \boldsymbol{\Delta} \hat{\boldsymbol{\theta}}_{j}^{k}\right)^{2}
$$

\section{Dimension of the Equation Set}

The equations and variables in the objective functions Eqn. (5) are not all linearly independent. The equations are formed by unit dual quaternions $\hat{Q}=\hat{q}+\varepsilon \hat{q}^{0}$ which have a dimension of eight, but are subject to the two implicit constraints $\hat{q} \hat{q}^{*}=1$ and $\hat{q} \cdot \hat{q}^{0}=0$, which reduce the independent dimension of the dual quaternion to 6 . Similarly, only four of the six Plücker components of each line are independent as they are subject to the two implicit constraints $\|\mathbf{s}\|=1$ and $\mathbf{s} \cdot \mathbf{s}^{0}=0$. The number of variables $x$ and independent variables $x^{0}$ can be written as,

$$
\begin{aligned}
x & =n(6+(m-1)) \\
x^{0} & =n(4+(m-1))
\end{aligned}
$$

The number of equations $f$ and independent equations $f^{0}$ can be written as,

$$
\begin{aligned}
f & =8 b(m-1) \\
f^{0} & =6 b(m-1)
\end{aligned}
$$

An important question remaining is how many task positions $m$ are required for the system to have a finite number of solutions, see [20] for details. This can be obtained by imposing $f^{0}=x^{0}$ and solving to obtain,

$$
m=\frac{4 n}{6 b-n}+1
$$

The number of task positions obtained is the amount needed to have a finite solution for non-degenerate tree topologies. Nondegenerate tree topologies are those where no kinematic chain segment, that is the smallest kinematic serial chain from an endeffector to a fork or from the origin to a fork, has fewer than 6 degrees-of-freedom and that no serial chain from the origin to an end effector has fewer joints than the largest kinematic chain segment from a fork to an end-effector. Degenerate tree topologies have redundant equations and thus Eqn. (10) is no longer valid.

More task positions can be provided although this overdetermines the system. If the system is overdetermined by task positions from the same workspace it will still have solutions, otherwise an exact match will generally not exist. Overdetermined systems can lead to problems of convergence and much slower performance.

Applying Eqn. (11) to our model with $b=5$ branches and $n=26$ revolute joints, three of them being shared by all the branches, we obtain that we can solve exactly for a task defined by $m=27$ finite positions for each finger. This gives a total of 156 structural parameters and 676 joint variables for our set of equation.

\section{Solver Implementation}

Note that the complexity of the problem that we are trying to solve is especially challenging. However, the use of Clifford algebra allows reducing the number of equations and variables, compared to a matrix-based approach. In particular, we represent the entire equation system with only 832 input variables between both structural and joint variables. This is a reduction of $33 \%$ in the number of variables compared to using homogeneous matrix, only considering the $3 \times 4$ submatrix containing the rotation and translation information.

Genetic algorithms have already been used in many kinematic problems $[21,22]$. The genetic algorithm performs an exhaustive exploration of the solution space in order find a solution. However, due to complexity of the system a pure genetic algorithm would have convergence problems past a certain fitness. This problem was overcome by reducing the solution space used by the genetic algorithm to only the local minima. This converts the solution space from a continuous domain to a discrete finite domain that improves the behaviour of the genetic algorithm. The local minima are found by a Levenberg-Marquardt 
optimizer.

The genetic algorithm chromosomes consist of sets of variables that belong to the full solution space. After being generated they are then converged to a local minima. This local optimization is also computed when chromosomes are crossed or mutated to ensure each chromosome always represents a local minima of the search space.

The fitness is calculated as the inverse of the sum of the error as seen in Eqn. (7). This makes the fitness a continuous positive function, which allows the usage of roulette-wheel selection when choosing pairs from the genetic algorithm population to crossover. It also converts the genetic algorithm to a maximization problem. The crossover rate is kept low to encourage diversity in the population, since the strong convergence is provided by the Levenberg-Marquardt optimizer.

To avoid explicit constraints the chromosomes are generated in the proximity of the ideal kinematic solution. This plays the role of a soft limit for the possible shapes of the robotic hand. However, it is not unusual for the Levenberg-Marquardt minimizer to move far from the generation space and find extremely non-anthropomorphic solutions.

The solver is executed until the fitness surpasses the value of $10^{10}$ at which it is considered to have arrived to a solution. The error is attributed to the imprecision in the computer representation of real numbers.

\section{Kinematic Subsystems}

It is worth noting that it is possible to solve a subsystem of the full kinematic chain tree. These subsystems are independently solvable as long as they have a finite number of task positions $m$ needed for a finite number of solutions. To have $m \in \mathbb{Q}^{+}$ the subsystem must satisfy the following inequality,

$$
6 b-r>0
$$

which is obtained from imposing $m>0$ in Eqn. (11). Table 2 shows all the possible subsystems of the hand model that can be solved.

The most interesting configurations to solve are the 5 kinematic chains at once, to minimize the needed positions, and the configuration with only two 4R branches to reduce the amount of variables needed. The two $4 \mathrm{R}$ branches are the index and middle fingers and can be seen in Fig. 3. Afterwards, the remaining fingers can be solved individually as the common revolute joints are now identified. There are also other systems that can be solved that are a compromise between the number of positions and the number of variables needed.

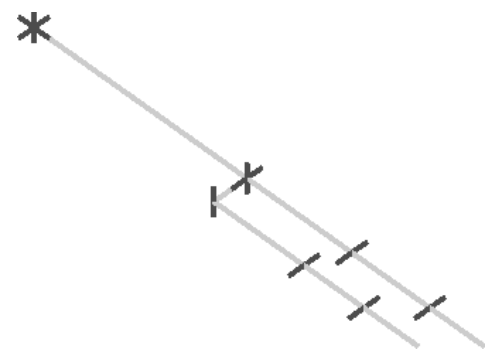

FIGURE 3: TOPOLOGY OF THE SOLVABLE (4R, 4R) FINGERS SUBSYSTEM.

TABLE 2: SOLVABLE SYSTEMS OF EQUATIONS FOR DIFFERENT COMBINATIONS OF CHAINS.

\begin{tabular}{cccccl}
\hline$b$ & $r$ & $x$ & $f$ & $m$ & Notes \\
\hline 5 & 26 & 832 & 1092 & 27 & Full model \\
4 & 22 & 1100 & 1452 & 45 & $(5 \mathrm{R}, 5 \mathrm{R}, 5 \mathrm{R}, 4 \mathrm{R})$ fingers \\
4 & 21 & 714 & 938 & 29 & $(5 \mathrm{R}, 5 \mathrm{R}, 4 \mathrm{R}, 4 \mathrm{R})$ fingers \\
3 & 17 & 1258 & 1666 & 69 & $(5 \mathrm{R}, 5 \mathrm{R}, 4 \mathrm{R})$ fingers \\
3 & 16 & 608 & 800 & 33 & $(5 \mathrm{R}, 4 \mathrm{R}, 4 \mathrm{R})$ fingers \\
2 & 11 & 550 & 726 & 45 & $(4 \mathrm{R}, 4 \mathrm{R})$ fingers \\
1 & 5 & 130 & 170 & 21 & 5R finger, common solved \\
1 & 4 & 56 & 72 & 9 & 4R finger, common solved \\
\hline
\end{tabular}

\section{RESULTS}

It is very important for the solver to converge that the endeffector positions, for all branches describing the task, do not follow an equation for the movement. This can lead to implicit equations that can lower the dimension of the system so that Eqn. (11) no longer guarantees a finite number of solutions. It is also important for the difference between end-effector positions to be large to aid in the solver convergence, and that all the joints present movement in the generated task.

The algorithm presented in this paper has a parallel nature and can be adjusted to run on supercomputers or other distributed computing systems to increase the calculation speed. This is due to the nature of the genetic algorithm which forms part of the hybrid solver presented.

We have obtained best results when using a population size of 100 for the genetic algorithm. This is much lower than what would be expected to be needed for a pure genetic algorithm for an equation system of this size. The crossover rate was set at 0.2 while the mutation rate was set extremely high at 0.5 . This is 
TABLE 3: SOLVER EXECUTION INFORMATION.

\begin{tabular}{ccc}
\hline Solution & Generations & Time (hours) \\
\hline 1 & 13 & 20.8 \\
2 & 5 & 20.5 \\
3 & 10 & 16.5 \\
4 & 12 & 85.0 \\
\hline
\end{tabular}

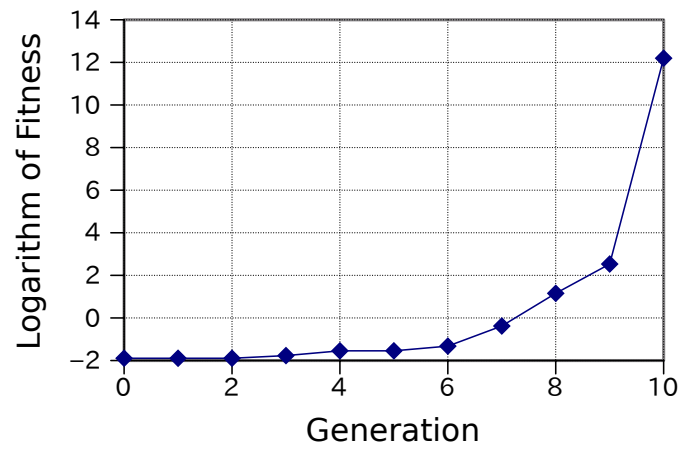

FIGURE 4: CONVERGENCE OF A SOLVER EXECUTION.

due to the fact that our hybrid solver uses a Levenberg-Marquadt optimizer which provides the strong convergence. Therefore the genetic algorithm must explore as much of the solution space as possible and not focus on local convergence.

Runtime information can be found in Table 3 for the various solutions found. These results were obtained on an Intel ${ }^{\circledR}$ Core $^{\mathrm{TM}}$ i7-870 CPU running at $2.93 \mathrm{GHz}$. The solver was using all 4 cores of the CPU by using 5 threads for the local optimization of the chromosomes, which is the slowest part of the algorithm. An example of the solver's converge can be seen in Fig. 4.

We present two example solutions that can be seen in Fig. 5 and Fig. 6. The non-anthropomorphic solutions are rendered on top of the anthropomorphic skeleton used to generate the data, and both are drawn as joint axes linked along their common normal lines. The anthropomorphic skeleton is represented by thick gray lines while the non-anthropomorphic solution is represented using thinner green lines.

\section{CONCLUSIONS}

In this paper we have presented a method for the kinematic synthesis of tree-like articulated systems, with an application in the design of a robot to perform human-hand tasks. To our knowledge this is the first time kinematic synthesis has been applied to tree topologies. This methodology allows obtaining nonanthropomorphic designs that can perform an anthropomorphic finite-position task exactly, while having a very different joint distribution and motion. These designs have applications in exoskeleton design, as they could be mounted on the hand without physically interfering with it.

The presented methodology is not limited to anthropomorphic tasks and hand models. It can be applied to generic serial, loop and tree topologies of articulated systems, making the solver a powerful tool.

Our hybrid solver has managed to successfully find solutions to kinematic synthesis problems much larger than previously considered. This has been accomplished by using Clifford algebra to provide a more compact system of equations that is then solved by the application of both meta-heuristics and classical math optimization.

From the dimensional synthesis point of view, an interesting result is that, due to the tree structure, we can perform exact synthesis for general serial chains with more than five degrees of freedom. We may conclude from this that, despite common believe that the human wrist/hand is a redundant mechanical system, it may not be so when we consider a task in which several fingers must act.

The dimensional synthesis for articulated systems like the one presented here, with a high number of joints, yields many solutions. A good selection process is required in order to choose a solution from the pool of candidates. For instance, for the application presented in this paper, additional constraints could be imposed either in the solving process or in the post-processing phase in order to find a suitable design. Among others, we can cite size or location restrictions for exoskeletons mounted on the human hand, or dexterity conditions at given configurations for manipulation in human environments. Future work will take into consideration possible link collisions that may be present in some of the solutions of the kinematic synthesis.

In order for the designs to be able to perform realistic human tasks, not only fingertip positions, but also fingertip forces and infinitesimal motion need to be considered. Future work will focus on including task velocities and accelerations in order to define grasping actions.

\section{ACKNOWLEDGMENT}

This work is partially supported by the Spanish Ministry of Science and Innovation under projects DPI2010-18449, Consolider Ingenio 2010 CSD2007-00018, and a Ramon y Cajal Research Fellowship. Also by the EU project GARNICS FP7247947. 


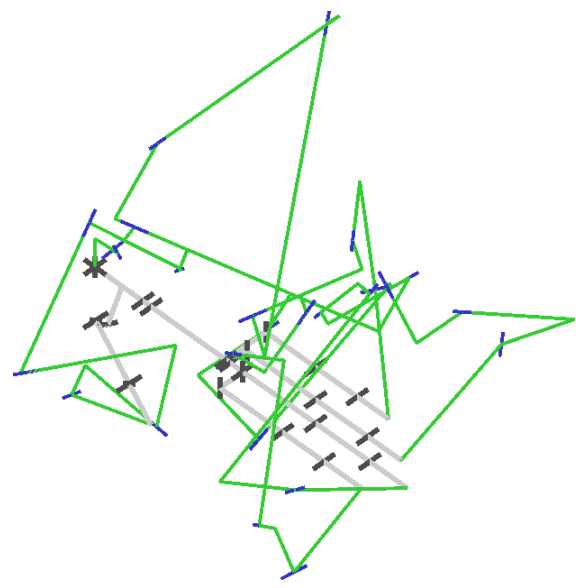

(a) Full Hand

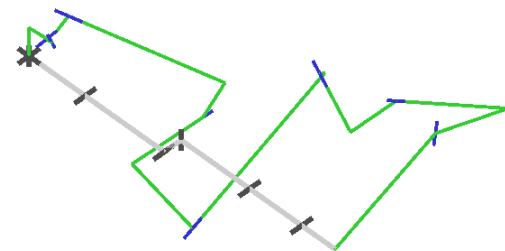

(d) Third Finger

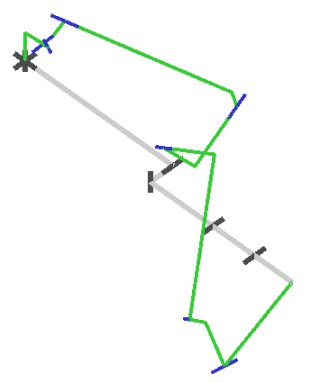

(b) Index Finger

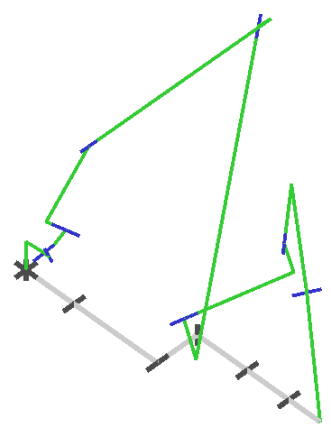

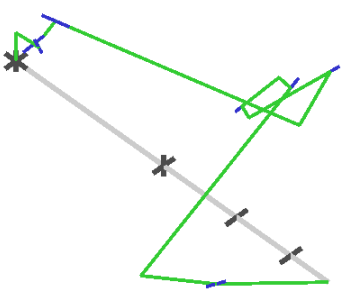

(c) Middle Finger

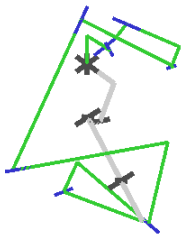

(e) Fourth Finger

(f) Thumb

FIGURE 5: OVERVIEW OF A SOLUTION FOUND.

\section{REFERENCES}

[1] Siciliano, B., and Khatib, O., 2008. Handbook of Robotics. Springer, Berlin.

[2] Soto Martell, J., and Gini, G., 2007. "Robotic hands: Design review and proposal of new design process". World Academy of Science, Engineering and Technology, 26.

[3] Dollar, A., and Howe, R., 2007. "Simple, robust autonomous grasping in unstructured environments". In Proc. of the 2007 International Conference on Robotics and Automation, pp. 4693-4700.

[4] Ciocarlie, A., and Allen, P., 2010. "Data-driven optimization for underactuated robotic hands". In Proc. of the 2010 International Conference on Robotics and Automation, pp. 1292-1299.

[5] Briglen, L., Laliberte, T., and Gosselin, C., 2008. Underactuated Robotic Hands. Springer.
[6] Biagiotti, L., Lotti, F., Melchiorri, C., and Vassura, G., 2004. How far is the human hand? a review of anthropomorphic robotic end-effectors. internal report. Tech. rep., Universita di Bologna.

[7] Dai, J., and Wang, D., 2007. "Geometric analysis and synthesis of the metamorphic robotic hand". ASME Journal of Mechanical Design, 129, pp. 1191-1197.

[8] van Varseveld, R., and Bone, G., 1999. "Design and implementation of a lightweight, large workspace nonanthropomorphic dexterous hand". ASME Journal of Mechanical Design, 121.

[9] Ramos, A., Gravagne, I., and Walker, I., 1999. "Goldfinger: A non-anthropomorphic, dextrous robot hand". In Proceedings of the 1999 IEEE International Conference on Robotics and Automation.

[10] Schaffer, C., and Dillmann, R., 2001. "Kinematic design 


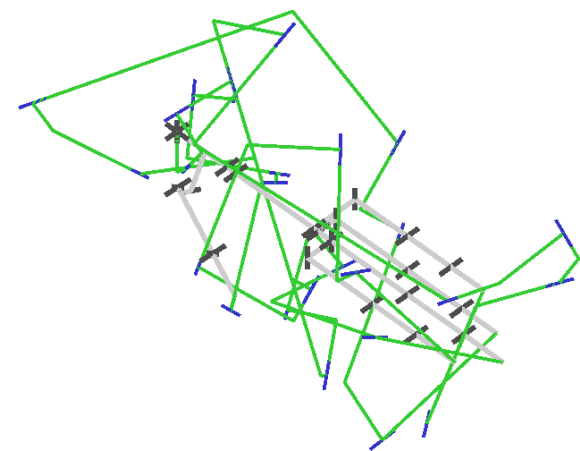

(a) Full Hand

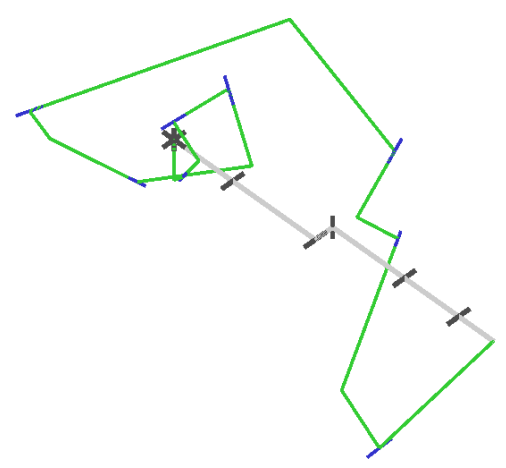

(d) Third Finger

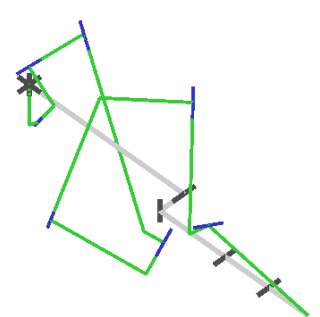

(b) Index Finger

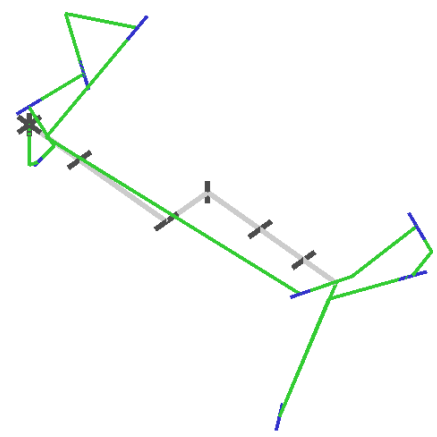

(e) Fourth Finger

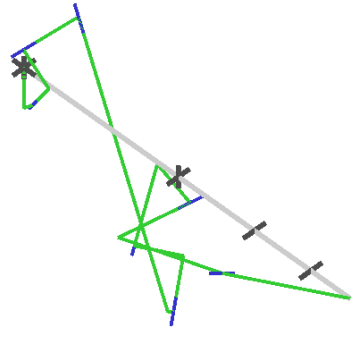

(c) Middle Finger

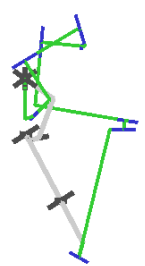

(f) Thumb

FIGURE 6: OVERVIEW OF A SECOND SOLUTION FOUND.

of a humanoid robot wrist". Journal of Robotic Systems, 18(12), pp. 747-754.

[11] Perez Gracia, A., and McCarthy, J. M., 2006. "The kinematic synthesis of spatial serial chains using clifford algebra exponentials". Proceedings of the Institution of Mechanical Engineers, Part C, Journal of Mechanical Engineering Science, 220(7), pp. 953-968.

[12] Perez, A., and McCarthy, J. M., 2003. "Dimensional synthesis of bennett linkages". ASME Journal of Mechanical Design, 125(1), pp. 98-104.

[13] Brunnthaler, K., 2006. "Synthesis of 4r linkages using kinematic mapping". $\mathrm{PhD}$ thesis, Institute for Basic Sciences and Engineering, University of Innsbruck, Innsbruck, Austria.

[14] Su, H., McCarthy, J. M., and Watson, L. T., 2004. “Generalized linear product homotopy algorithms and the computation of reachable surfaces". ASME Journal of Computers and Information Science and Engineering, 4(3), pp. 226235.

[15] Lee, E., Mavroidis, C., and Merlet, J. P., 2004. "Five precision point synthesis of spatial rrr manipulators using interval analysis". ASME Journal of Mechanical Design,
126(5), pp. 842-850.

[16] Fadi J. Beijjani, J. M. F. L., 1989. Basic Biomechanics of the Musculoskeletal System. Lea \& Febiger, ch. Biomechanics of the hand, pp. 275-304.

[17] Chang, L., and Matsuoka, Y., 2006. "A kinematic thumb model for the act hand". In Robotics and Automation, 2006. ICRA 2006. Proceedings 2006 IEEE International Conference on, pp. $1000-1005$.

[18] Lee, E., and Mavroidis, C., 2002. "Solving the geometric design problem of spatial $3 r$ robot manipulators using polynomial homotopy continuation". ASME Journal of Mechanical Design, 124(4), pp. 652-661.

[19] Murray, R. M., Li, Z., and Sastry, S. S., 1994. A Mathematical Introduction to Robotic Manipulation. CRC Press, Inc., Boca Raton, FL.

[20] Perez, A., and McCarthy, J. M., 2004. "Dual quaternion synthesis of constrained robotic systems". ASME Journal of Mechanical Design, 126(3), pp. 425-435.

[21] Chocron, O., and Bidaud, P., 1997. "Evolutionary algorithms in kinematic design of robotic systems". In Intelligent Robots and Systems, 1997. IROS '97., Proceedings of the 1997 IEEE/RSJ International Conference on, Vol. 2, 
pp. $1111-1117$ vol.2.

[22] Yang, Y., Peng, G., Wang, Y., and Zhang, H., 2007. “A new solution for inverse kinematics of 7-dof manipulator based on genetic algorithm". In Automation and Logistics, 2007 IEEE International Conference on, pp. 1947 -1951. 